\title{
Reflow of obstructed capillaries in the maculae of humans with diabetic retinopathy, observed by fluorescein angiography
}

\author{
YASUO YAMANA, ${ }^{2}$ YOSHISUKE OKA,' YOSHITAKA OHNISHI, \\ TATSURO ISHIBASHI ${ }^{2}$ AND TOYOSHI INOGUCHI
}

From 'Ohshima Eye Hospital; the ${ }^{2}$ Department of Ophthalmology, Faculty of Medicine, Kyushu University; and the ${ }^{3}$ Third Department of Internal Medicine, Faculty of Medicine, Kyushu University, Japan

SUMMARY As diabetic retinopathy progresses, the non-perfused area in the retina gradually enlarges as the capillaries are obstructed. To elucidate the process of capillary obstruction we examined macular capillaries in 104 diabetics, using fluorescein angiography. The follow-up period was three to eight years. We found that some capillaries in the macula were obstructed, then became unobstructed. Reflow of blood into the macula after the obstruction was observed in nine patients $(8 \cdot 7 \%)$. The time between obstruction and reflow varied from two months to four years. There were four cases of simple, three of preproliferative, and two of proliferative retinopathy. Reflow in the capillaries was confirmed as occurring at the same position and in vessels with the same diameter as seen before the obstruction. There was no leakage of the fluorescein dye from the capillary where reflow took place. We tentatively conclude that one of the causes of this phenomenon is a functional obstruction.

It is generally accepted that diabetic retinopathy is a form of microangiopathy. As the retinopathy progresses, the non-perfused area in the retina gradually enlarges as a result of obstruction of the capillaries.

Although several ophthalmologists reported this phenomenon, each report was of one case and the state of the capillaries before the obstruction was not described. ${ }^{1-5}$ Almost all reported that this phenomenon occurred after photocoagulation. We therefore examined the occurrence rate of the reflow of the obstructed capillaries, the type of capillary reflow, and the possible relation to photocoagulation.

\section{Subjects and methods}

Fluorescein angiography was carried out from 1977 to 1985 on 104 eyes of 104 diabetics aged 16 to 78 years. All were treated by diabetologists and the follow-up was for three to eight years. Thirty were cases of insulin-dependent diabetes mellitus (IDDM) and 74 of non-insulin-dependent diabetes mellitus (NIDDM). Diabetics without retinopathy, deter-

Correspondence to Yasuo Yamana, MD. Yamana Eye Clinic, 10-Nabeyama Nakama-shi Fukuoka, 809 Japan. mined by ophthalmoscopy, numbered 22 . In 58 cases there was a simple retinopathy, 18 had a preproliferative retinopathy, and six a proliferative retinopathy.

All patients were examined by ophthalmoscopy after the pupil had been dilated with $10 \%$ phenylephrine and $0.5 \%$ cyclopentolate. Fluorescein fundus angiography and colour photography were carried out periodically, the frequency depending on the severity of the diabetic retinopathy. $5 \mathrm{ml}$ of $10 \%$ sodium fluorescein was given intravenously.

We examined coagulation and platelet aggregation at the time of occlusion, and adenosine-5'diphosphate (ADP) and coagulation were examined at the time of occlusion and after reflow in patient 1 and after reflow in eight patients.

\section{Results}

Reflow of blood into the macular capillary bed after the obstruction occurred in nine of the 104 diabetics $(8.7 \%)$. Table 1 is a summary of data on nine with a reflow in the obstructed capillaries. Five were cases of IDDM and four of NIDDM. The ages of the patients at the time of reflow ranged from 19 to 53 
Table 1 Summary of nine cases

\begin{tabular}{|c|c|c|c|c|c|c|c|c|c|}
\hline Patients & 1 & 2 & 3 & 4 & 5 & 6 & 7 & 8 & 9 \\
\hline Age & 46 & 48 & 30 & 53 & 19 & 35 & 43 & 34 & 46 \\
\hline Sex & Female & Female & Female & Female & Female & Male & Male & Male & Male \\
\hline Type of diabetes & NIDDM & NIDDM & IDDM & NIDDM & IDDM & IDDM & IDDM & IDDM & NIDDM \\
\hline Duration of diabetes $(\mathrm{yr})$ & 5 & 10 & 11 & 11 & 12 & 13 & 16 & 17 & 22 \\
\hline Photocoagulation & - & - & - & - & - & - & + & - & + \\
\hline Control of blood sugar & Poor & Poor & Poor & Poor & Fair & Poor & Poor & Poor & Poor \\
\hline Other complications & - & - & - & - & 一 & - & $\mathrm{Np}$ & $\mathrm{Np}, \mathrm{Nr}$ & - \\
\hline \multicolumn{10}{|l|}{ Visual acuity } \\
\hline before occlusion & 0.9 & $0 \cdot 1$ & 0.8 & 0.9 & 0.8 & $1 \cdot 0$ & $0 \cdot 8$ & $1 \cdot 0$ & 0.4 \\
\hline after occlusion & 0.7 & $0 \cdot 1$ & $0 \cdot 8$ & 0.7 & $1 \cdot 2$ & $0 \cdot 6$ & 0.7 & $0 \cdot 6$ & $0 \cdot 7$ \\
\hline \multicolumn{10}{|l|}{ Retinopathy } \\
\hline before occlusion & $\mathbf{S}$ & PP & $\mathbf{S}$ & $\mathbf{S}$ & $\mathbf{S}$ & $\mathbf{S}$ & PP & PP & $\mathbf{P}$ \\
\hline after occlusion & PP & PP & $\mathbf{S}$ & $\mathbf{S}$ & $\mathbf{S}$ & $\mathbf{S}$ & PP & $\mathbf{P}$ & $\mathbf{P}$ \\
\hline
\end{tabular}

Np: nephropathy. Nr: neuropathy. S: simple retinopathy. PP: preproliferative retinopathy. P: proliferative retinopathy.

years. There were four men and five women. The duration of diabetes was over 10 years in all cases except one, in which it was 5 years. Blood sugar control was poor except for one case, where it was fair; however, there was no history of hypoglycaemic attack. Visual acuity ranged from 0.1 to 1.0 at the time of occlusion and after reflow in nine cases. Visual acuity worsened in five, improved in two, and was unchanged in two. One had nephropathy, and another nephropathy and neuropathy. Photocoagulation was performed on 32 of the 104 diabetics and on two of the nine cases with reflow.

Table 2 shows data on the coagulation of nine patients. Data after reflow showed increased platelet coagulation activity. Data on patient 1 showed an increase in platelet coagulation activity both during occlusion and after reflow of the capillary.

\section{Case reports}

CASE 6

A 35-year-old man (Figs. 1-3) had had IDDM for seven years. His right eye showed a simple retinopathy. Fig. 1 shows colour fundus photographs (in black-and-white) and fig. 2 fluorescein angiographs, at the late stage. The upper photographs 'a' of Figs. 1 and 2 indicate the time before obstruction, the middle photographs ' $b$ ' indicate the time of obstruction, and the lower ones 'c' the time after the reflow. These photographs demonstrate a gradual progression of simple retinopathy. Fig. 3 shows enlarged angiographs of the macular area in this patient. The capillary indicated by the arrow is the normal angiogram in photograph 'a'.

Two years and eight months later that capillary was absent in photograph ' $b$ '. This occluded capillary was not demonstrated from the early capillary phase to the late phase. However, two years and 10 months after the time of ' $b$ ' there was a reflow in photograph ' $c$ ' at the same position and with the same diameter as in photograph ' $a$ '. There was no leakage of the fluorescein dye from the capillary, as indicated by the arrow. Damage of other capillaries of the surrounding fovea progressed from the time of ' $a$ ' to the time of ' $b$ '.

\section{CASE 8}

A 34-year-old man (Figs. 4-6) had had IDDM for 12 years. His right eye showed a preproliferative retinopathy at the time of ' $a$ ' and progressed to proliferative

Table 2 Coagulation

\begin{tabular}{|c|c|c|c|c|c|c|c|c|c|c|}
\hline Patients & 1 & 2 & 3 & 4 & 5 & 6 & 7 & 8 & 9 & Control \\
\hline \multicolumn{11}{|l|}{ Platelet aggregation } \\
\hline Factor IV (ng/ml) & & & & & & & & & & $\leqslant 20 \mu \mathrm{g} / 1$ \\
\hline after reflow & 30 & $100 \uparrow$ & 63 & $100 \uparrow$ & 85 & 30 & 50 & 78 & 80 & \multirow{5}{*}{$\begin{array}{l}\leqslant 50 \mu \mathrm{g} / \mathrm{l} \\
\leqslant 56 \%(2 \mu \mathrm{mol})\end{array}$} \\
\hline $\begin{array}{l}\beta \text {-thromboglobulin }(\mathrm{ng} / \mathrm{ml}) \\
\text { after reflow }\end{array}$ & & & & & & & & & & \\
\hline $\begin{array}{l}\text { after reflow } \\
\operatorname{ADP}(\%)\end{array}$ & 110 & $200 \uparrow$ & 150 & 170 & 170 & 110 & 99 & 150 & $200 \uparrow$ & \\
\hline before occlusion & 61 & - & - & - & - & - & - & - & - & \\
\hline after reflow & 61 & 46 & 64 & 59 & 48 & 72 & 52 & 64 & - & \\
\hline \multirow{3}{*}{$\begin{array}{l}\text { Collagen (\%) } \\
\text { before occlusion } \\
\text { after reflow }\end{array}$} & & & & & & & & & & \multirow[t]{3}{*}{$\leqslant 66 \%(1 \mathrm{mg} / \mathrm{l})$} \\
\hline & 97 & - & - & - & - & 一 & - & - & - & \\
\hline & 97 & 64 & 79 & 89 & 58 & 86 & 48 & 78 & - & \\
\hline
\end{tabular}




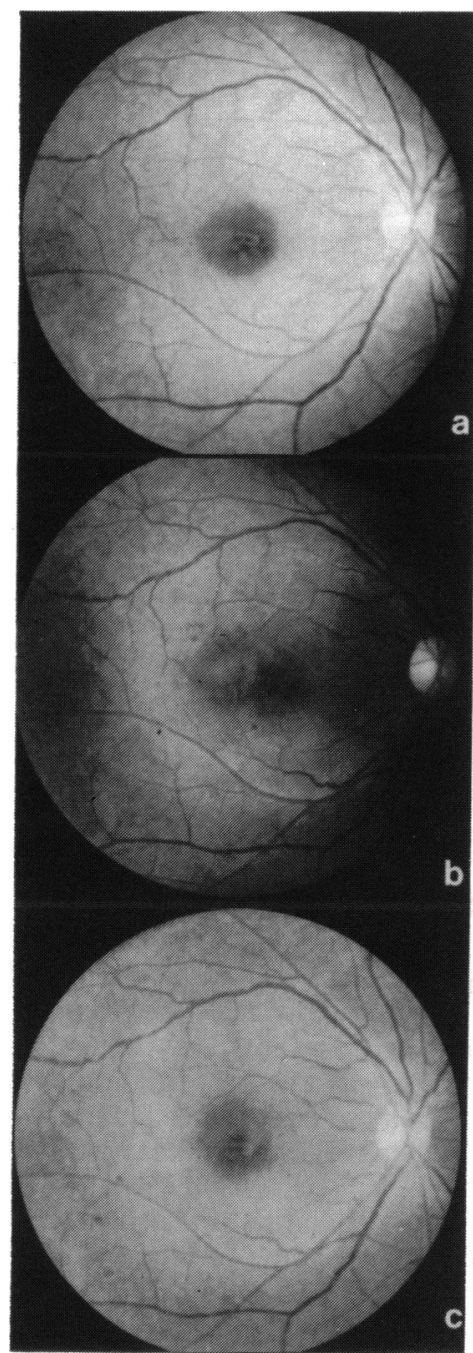

Fig. 1 Case 6. Fundus photographs of a 35-year-old man who had had IDDM for seven years. His right eye showed a simple retinopathy. Figs. $1 a, 2 a, 3$ a were taken at the time before obstruction, Figs. $1 b, 2 b, 3 b$ at the time of obstruction, and Figs. 1c, $2 c, 3 c$ after the reflow.

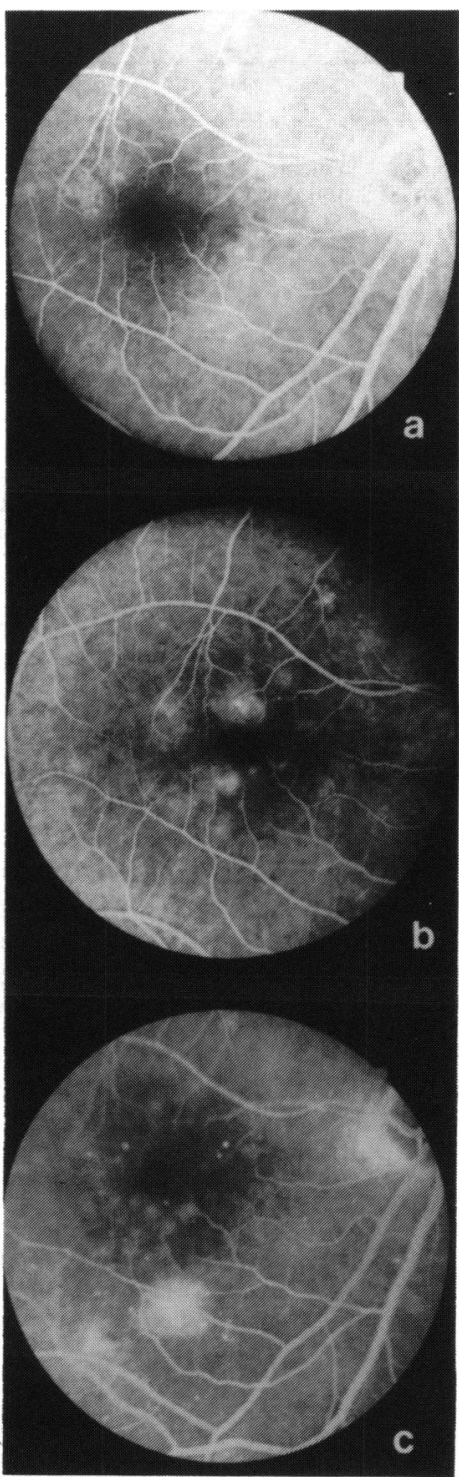

Fig. 2 Fluorescein angiographs of Fig. 1 at the late stage. A gradual progression in simple retinopathy is evident. retinopathy at the time of 'c' (Figs. 4-5). Fig. 6 shows enlarged angiographs of the macular area. The capillary indicated by the arrow is the normal angiogram in photograph ' $a$ '. This same capillary in photograph ' $b$ ' is not evident four years and 10 months later. This occluded capillary was not visible from the early capillary phase to the late phase. However, three months after the time of ' $b$ ' there was evidence of a reflow in photograph ' $c$ ', at the same position and with the same diameter as seen in photograph ' $a$ '. There was no leakage of the fluorescein dye from the capillary, as indicated by the arrow.

\section{CASE 2}

A 48-year-old woman (Figs. 7-9) had had NIDDM for nine years. Her right eye showed a preproliferative retinopathy and this state gradually worsened
Fig. 3 Enlarged angiographs of the macular area of the case 6 . The capillary indicated by the arrow shows the normal angiogram in photograph $\mathrm{a}$. Two years and eight months later this same capillary cannot be seen in photograph $\mathrm{b}$. However, two years and 10 months after the time of $\mathrm{b}$ there is a reflow, in photograph c, at the same position and with the same diameter as seen in photograph a. There is no leakage of the fluorescein dye, as indicated by the arrow. 


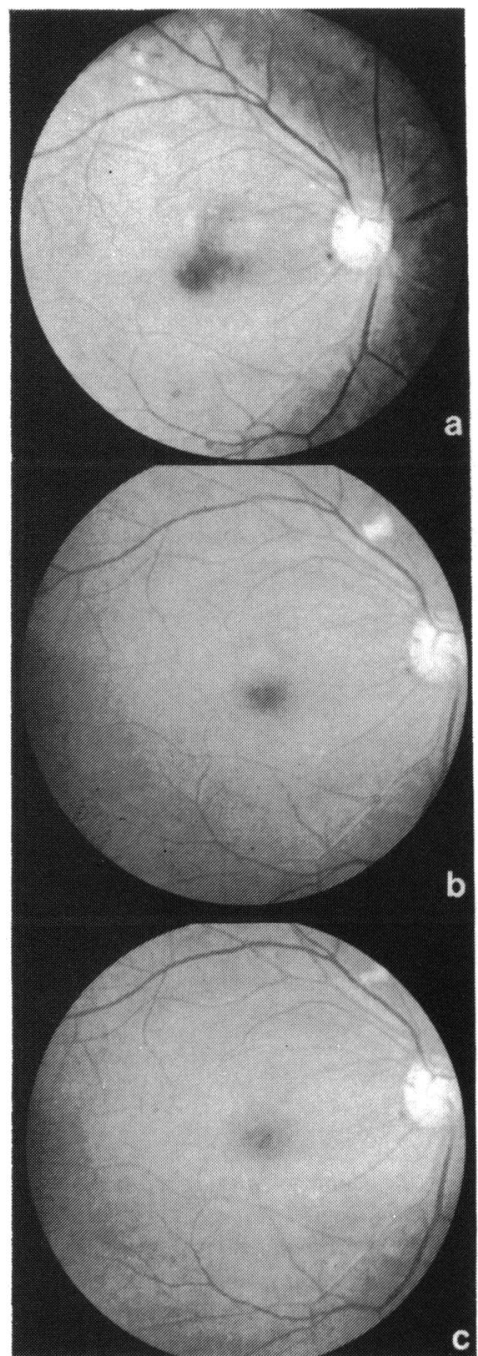

Fig. 4 Case 8, a 34-year-old man who had had IDDM for 12 years. Figs. $4 a$, $5 a, 6 a$ were taken at the time before obstruction, Figs. $4 b, 5 b, 6 b$ at the time of obstruction, and Figs. $4 c, 5 c, 6 c$ at the time after the reflow. His right eye showed preproliferative retinopathy in the upper photo a and progressed to proliferative retinopathy in the lower photo c.

(Figs. 7-8). Fig. 9 shows enlarged photographs of the macular area. The capillary indicated by the arrow is the normal angiogram in photograph ' $a$ '. Three years and eight months later that capillary was absent in photograph ' $b$ '. This occluded capillary was not angiographed at the late stage. However, two months after the time of ' $b$ ' the capillary was visible in

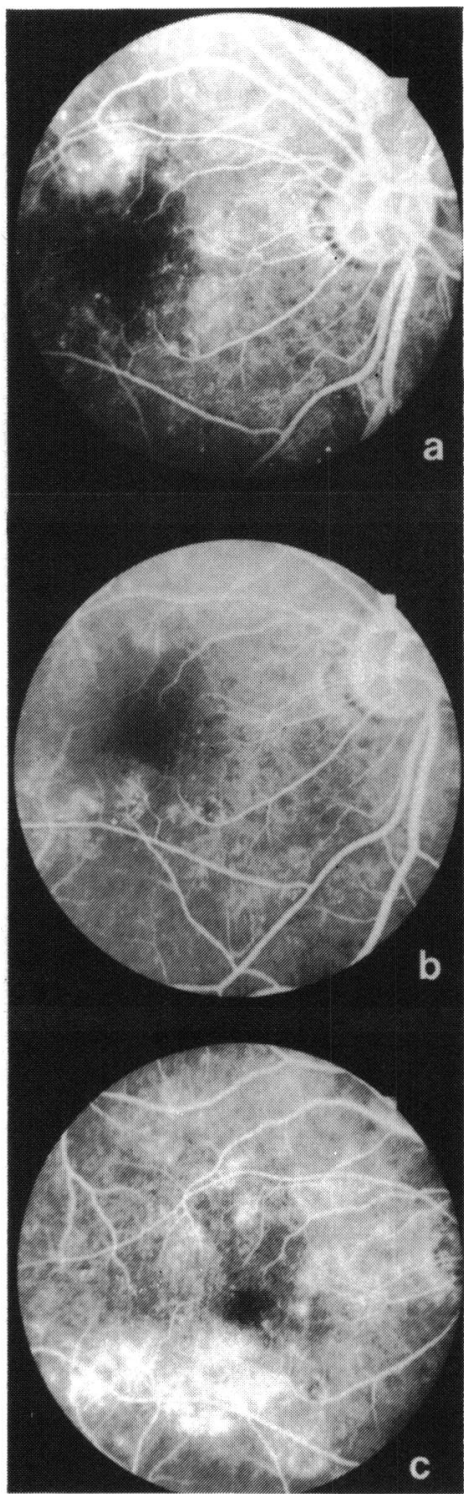

Fig. 5 Fluorescein angiographs of Fig. 4 at the late stage. There is mild leakage and progression of retinopathy in the macular area.

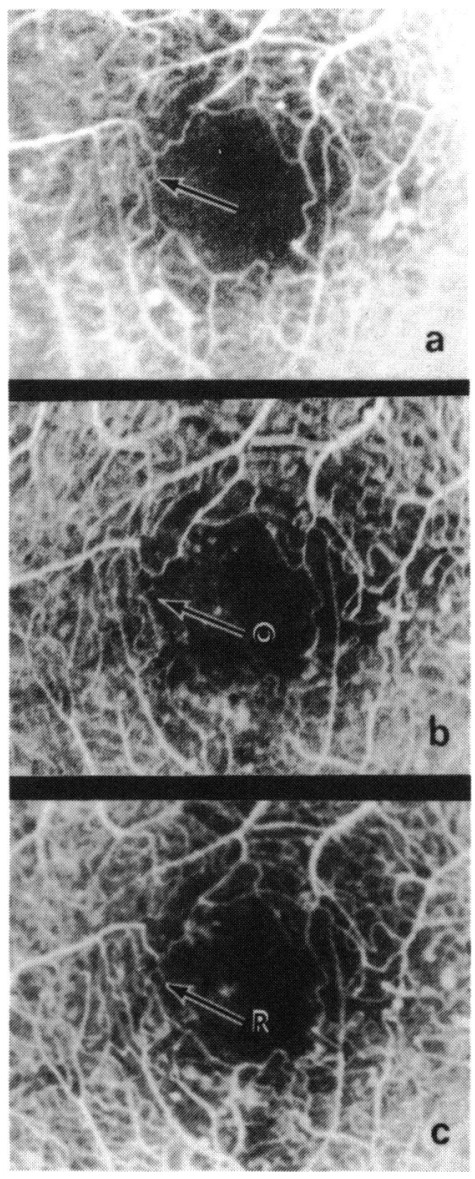

Fig. 6 Enlarged angiographs of the macular area of the case 8 . The capillary indicated by the arrow shows a normal angiogram, in photograph a. Four years and 10 months later that capillary cannot be seen in photograph b. However, three months after the time of b there is a reflow in photograph $\mathrm{c}$, at the same position and with the same diameter as seen in photograph a. There is no leakage of the fluorescein dye, as indicated by the arrow.

photograph 'c', at the same position and with the same diameter as seen in photograph ' $a$ '. There was no leakage from the capillary, as indicated by the arrow.

\section{Discussion}

It is generally accepted that, in vasculo-occlusive disease such as diabetic retinopathy, as the retino- 


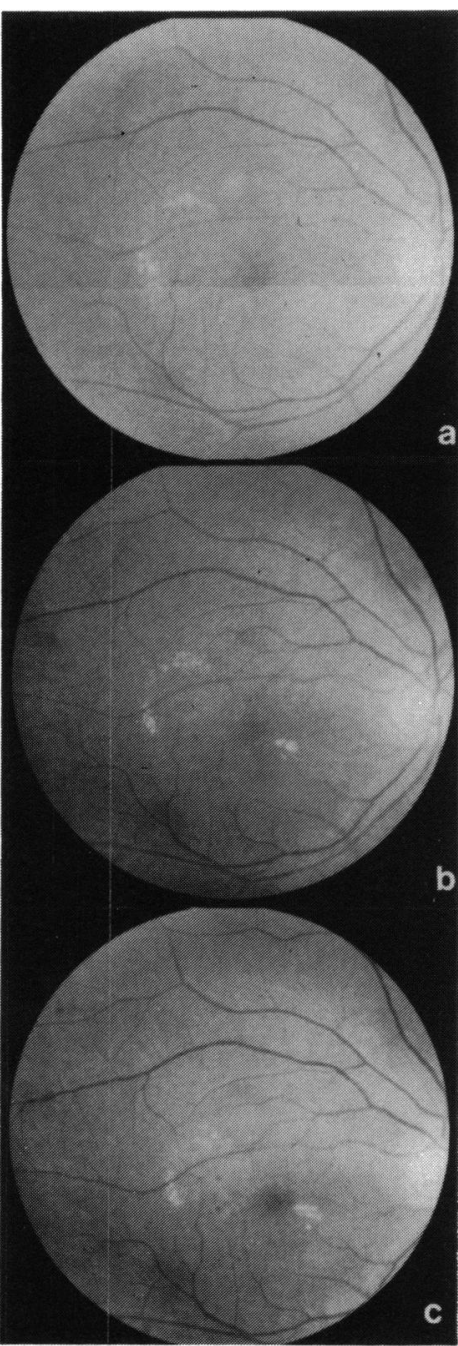

Fig. 7 Case 2, a 48-year-old woman who had had NIDDM for nine years. Figs. $7 a, 8 a, 9 a$ were taken at the time before obstruction, Figs. $7 b, 8 b, 9 b$ at the time of obstruction, and Figs. $7 c$, $8 c, 9 c$ at the time after the reflow. Her right eye showed preproliferative retinopathy in the upper photo a, and the retinopathy gradually progressed in the lower photo $\mathrm{c}$.

pathy progresses the non-perfused area in the retina gradually enlarges owing to obstruction of the capillaries. On the other hand there are reports on the reflow of blood into the capillary after obstruction in cases of retinal vein occlusion, ${ }^{6}$ Takayasu's disease, ${ }^{7}$ sickle cell retinopathy, ${ }^{8}$ and diabetic retinopathy. ${ }^{1-5}$ It was reported that recanalisation occurred after

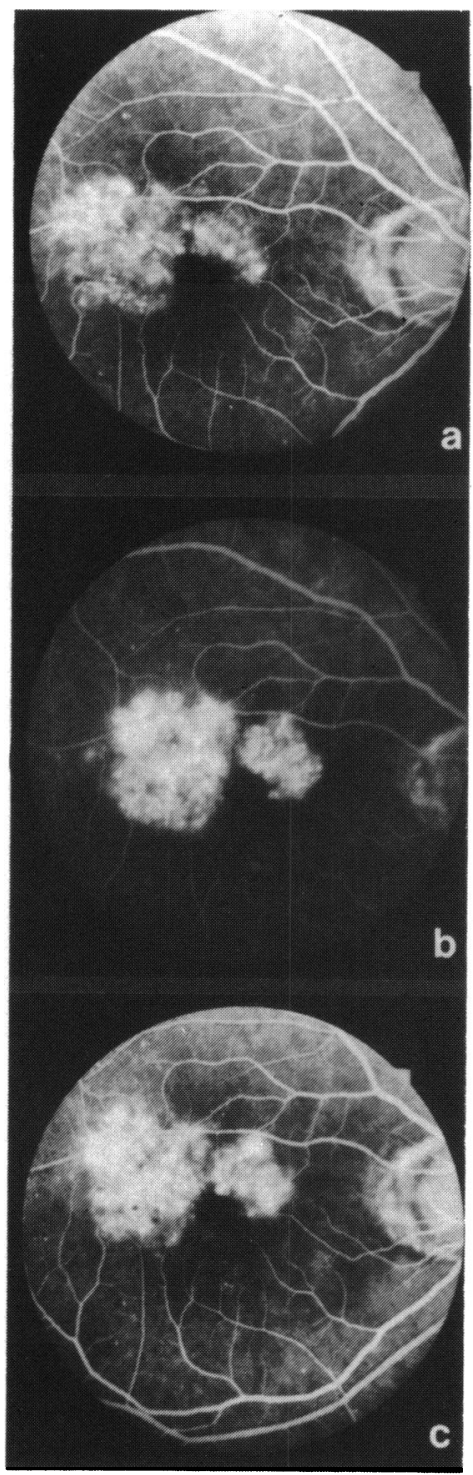

Fig. 8 Fluorescein angiographs of Fig. 7 at the late stage. There is extensive leakage and progression of retinopathy in the macular area.

a
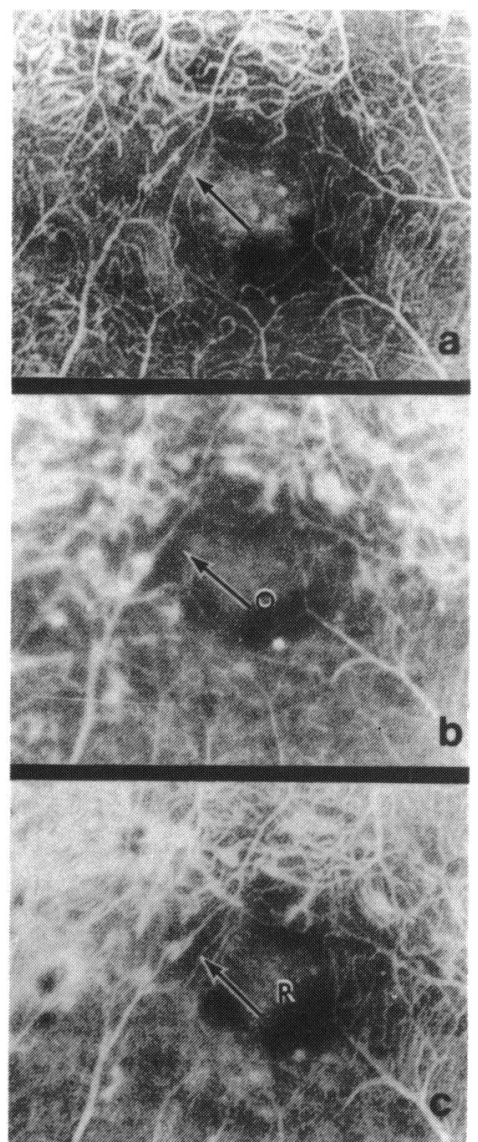

Fig. 9 Enlarged angiographs of macular area in the case 2 . The capillary indicated by the arrow shows the normal angiogram in photograph a. Three years and eight months later that capillary cannot be seen in photograph b. However, two months after the time of $\mathrm{b}$ there is a reflow in photograph $\mathrm{c}$, at the same position and with the same diameter as seen in photograph a.

There is no leakage of the fluorescein dye, indicated by the arrow.

photocoagulation, and capillaries were not visible before the obstruction.

It is necessary to distinguish a capillary with a reflow from a new vessel. We examined and followed up macular capillaries of diabetics, using fluorescein angiography, the objective being to elucidate the process of capillary obstruction. We observed capillaries before the obstruction, at the time of obstruction, and after the reflow of blood. These capillaries 
are at the same position and had the same diameter as before the obstruction. There was no leakage of fluorescein dye from the capillary. Therefore these capillaries are not new vessels and the obstructions of the capillaries are probably functional, not organic. The reflow of blood was not observed in cases of early diabetic retinopathy and cases without retinopathy by ophthalmoscopy and fluorescein angiography. ${ }^{9}$

There is well substantiated evidence that patients with diabetes and also experimental diabetic rats have abnormalities of platelet aggregation. ${ }^{111}$ The presence of a significant delay of systemic and intraocular circulation in diabetics, determined by fluorescein angiography, has been reported. ${ }^{12}$ Coagulation and platelet aggregation in our patients were accelerated not only at the time of occlusion but also after reflow, and in all of them the retinopathy progressed. This evidence indicates that capillary flow in diabetics with progressive retinopathy is sluggish or repeatedly ceases under conditions of acceleration of coagulation and platelet aggregation. One cause of this phenomenon is functional obstruction.

\section{References}

1 Muraoka K, Shimizu K. Intraretinal neovascularization in diabetic retinopathy. Ophthalmology 1984; 91: 1440-6.
2 Mohan R, Kohner EV. Retinal revascularisation in diabetic retinopathy. $\mathrm{Br} J$ Ophthalmol 1986; 70: 114-7.

3 Kohner EM, McLeod D, Marshall J. Diabetic disease. In: Keen $\mathrm{H}$, Jareet J, eds. Complications of diabetes. 2nd ed. London: Arnold, 1982: 19-108.

4 Krill AE, Archer DB, Newell FW, Chishti DB. Photocoagulation in diabetic retinopathy. Am J Ophthalmol 1971; 72: 299-321.

5 Okada M, Tamura T. Recanalization of retinal capillary nonperfusion in diabetic retinopathy following photocoagulation. A long-term follow up study. Rinsho Ganka 1984; 38: 1017-20.

6 Peduzzi M, De Rosa, Fonda S, Coccheri S. Haematostatic studies in retinal vein occlusion. Fibrinolytic response to venostasis as a prognostic factor for spontaneous recanalization. Thromb Res 1981; 24: 105-18.

7 Tanaka T, Henmi T, Awane H. Capillary nonperfusion and arteriovenous shunts in the retina in Takayasu's disease. Rinsho Ganka 1985; 39: 425-31.

8 Galinos SO, Asdourian GK, Woolf MB, et al. Spontaneous remodeling of the peripheral vasculature in sickling disorders. Am J Ophthalmol 1985; 79: 853-70.

9 Yamana Y, Ohnishi Y, Taniguchi Y, Ikeda M. Early signs of diabetic retinopathy by fluorescein angiopathy. Jpn $J$ Ophthalmol 1983; 27: 218-28.

10 Preston FE, Ward JD, Marcola BH, Porter NR, Timperley WR, O'Malley BC. Elevated beta-thromboglobulin levels and circulating platelet aggregation in diabetic microangiopathy. Lancet 1978; i: $238-40$.

11 Ishibashi $\mathrm{T}$, Tanaka $\mathrm{K}$, Taniguchi $\mathrm{Y}$. Platelet aggregation and coagulation in the pathogenesis of diabetic retinopathy in rats. Diabetes 1981; 30: 601-6.

12 Okano T. Fluorescein angiography in diabetic patients. Nippon Ganka Gakkai Zasshi 1977; 81: 69-134.

Accepted for publication 26 June 1987. 